\title{
Convex Representations of Maps on the Torus and Other Flat Surfaces*
}

\author{
Bojan Mohar \\ University of Ljubljana, Department of Mathematics, \\ Jadranska 19, 61111 Ljubljana, Slovenia \\ bojan.mohar@uni-lj.ac.mail.si
}

\begin{abstract}
It is shown that every map on the torus satisfying the obvious necessary conditions has a convex representation on the flat torus $R^{2} / Z^{2}$. The same holds for the Klein bottle, and the two bordered flat surfaces-the cylinder and the Möbius band. In each case, a linear-time algorithm is obtained for constructing convex representations if they exist.
\end{abstract}

\section{Flat Surfaces and Convex Maps}

By a well-known result of Stein [12] and Tutte [15] every 3-connected planar graph has a convex embedding in the plane, i.e., there is a representation of such a graph in the plane such that all bounded faces are (strictly) convex polygons and the unbounded face is a complement of a convex polygon. This result was further generalized by Thomassen [14] who discovered necessary and sufficient conditions for the existence of convex embeddings in the plane (see Section 3). A result which is closely related to the Stein-Tutte theorem is the theorem of Steinitz [13] that every 3-connected planar graph can be represented as the graph of a convex 3-polytope. There were attempts to generalize Steinitz's theorem to maps on surfaces of positive genus, e.g., [3] and [6]. However, it seems that no one tried to extend the Stein-Tutte theorem to nonsimply connected surfaces. In this paper we fill in this gap by proving a corresponding result for the torus, the Klein bottle, the cylinder, and the Möbius band. These are the only flat surfaces with straight boundary components. The existence of convex representations of maps on the torus and the Klein bottle has some important consequences since their universal

\footnotetext{
* This research was supported in part by the Ministry for Science and Technology of Slovenia, Research Project P1-0210-101-92.
} 
covers give rise to convex tilings of the Euclidean plane. A discussion about this can be found on p. 202 of [5].

Let $S$ be a compact surface. A map on $S$ is a pair $M=(G, S)$, where $G$ is a connected graph embedded in $S$ such that all faces are simply connected. Recall that a face of a map $M=(G, S)$ is a connected component of $S \backslash G$. Moreover, if $S$ has nonempty boundary, $\partial S \neq \varnothing$, then we require that $\partial S$ is covered by $E(G)$, i.e., for each point $p \in \partial S$ there is an edge of $G$ which contains $p$. Note that we do not require the underlying graph of a map to be simple, i.e., we allow loops and parallel edges. A map on the torus is also called a toroidal map. For a map $M=(G, S)$ we use $V(M), E(M)$, and other graph-theory notation and terminology to denote the corresponding quantities of the graph $G$ of the map. Two maps $M=(G, S)$ and $M^{\prime}=\left(G^{\prime}, S^{\prime}\right)$ are equivalent if there is a homeomorphism $h: S \rightarrow S^{\prime}$ mapping the graph $G$ of the first map isomorphically to the graph $G^{\prime}$ of the second map. It is well known (see, e.g., [4] and [10]) that two maps on an orientable surface without boundary are equivalent if and only if they determine the same rotation system on the graph.

A compact Riemannian surface $S$ (possibly with boundary) is flat if every point $p \in S$ has a neighborhood which is affinely diffeomorphic to an open set in the closed upper half-plane of the Euclidean plane. This is equivalent to the condition that the curvature and torsion are identically zero, including the curvature of the boundary $\partial S$ being zero. The special case of a flat surface is the flat torus, the quotient space $\mathbf{R}^{2} / \mathbf{Z}^{2}\left(\mathbf{R}^{2} / \sim\right.$ where $(x, y) \sim\left(x^{\prime}, y^{\prime}\right)$ means $\left(x-x^{\prime}, y-y^{\prime}\right)$ $\in \mathbf{Z}^{2}$ ). Another flat surface is the flat Klein bottle. This surface is the quotient of the Euclidean plane $\mathbf{R}^{2}$ corresponding to the relation $\sim$ given by $(x, y) \sim$ $\left(x+n,(-1)^{n} y+m\right), n, m \in \mathbf{Z}$. The flat torus and the flat Klein bottle are usually represented as the identification space of the unit square by identifying the top and the bottom side and then identifying the left and the right, with a previous turn by $180^{\circ}$ in the case of the Klein bottle. There are two additional flat surfaces with boundary - the flat cylinder and the flat Möbius band. They are obtained from the unit square as well, by identifying only one pair of sides, the left and the right. To get the cylinder, they are identified without a turn, and to get the Möbius band we perform a twist of $180^{\circ}$ of one side before the identification. It can be shown by using the Gauss-Bonnet formula (see [1]) that these are the only compact flat surfaces.

Let $M=(G, S)$ be a map. A face $F$ of $M$ is convex if for any two points $x, y \in F$ there is a geodesic contained in $F$ joining $x$ and $y$. The map $M$ is convex if all of its faces are convex and all edges of $G$ are geodesic segments. (It can be shown using the Gauss-Bonnet formula that on a surface with nonpositive curvature an interior edge must be geodesic if the two faces containing it are convex; but this does not necessarily hold for the edges on the boundary.) On a flat surface $S$, a face is convex if and only if it is bounded by straight line segments and each interior angle is smaller or equal to $\pi$.

Every convex map $M$ on a flat surface $S$ is reduced (see Section 2). Roughly speaking, it has the same property as 3-connected planar maps. It is shown in this paper that being reduced is also sufficient for a toroidal map to be equivalent to a convex map on the flat torus (Theorem 4.2). The same results are then shown 
for maps on other flat surfaces (Theorems 5.1, 6.1, and 6.2). As a corollary, we get necessary and sufficient conditions for a weaker property, the existence of straightline representations of maps on flat surfaces.

In addition to the existence of convex representations, we also present a linear-time algorithm to construct such an embedding, if it exists.

\section{Reduced Maps}

A map $M=(G, S)$ is reducible if it has one of the following properties:

(R1) $G$ contains a pendant edge (equivalently, $G$ has a vertex of degree 1).

(R2) $G$ contains a cycle $C$ which bounds a (closed) disk $D$ on $S$ such that there are at most two vertices of $C$ which are adjacent with an edge not in $D \cup C$.

(R3) In $G$ there are homotopic cycles $C_{1}$ and $C_{2}$ such that $C_{1} \cap C_{2}$ is a vertex, say $x \in V(G)$, and all vertices on $C_{1} \cup C_{2}$, except possibly $x$, have all their adjacent edges in $D \cup C_{1} \cup C_{2}$, where $D$ is the (open) disk bounded by $C_{1} \cup C_{2}$.

A nonreducible map is said to be reduced. The following proposition, whose easy proof is left to the reader, shows that reduced maps are some kind of a generalization of 3-connected planar maps to maps on general surfaces.

Proposition 2.1. Let $M=(G, S)$ be a map on a nonsimply connected surface $S$. If $G$ is 3-connected, then $M$ is reduced.

On the other hand there are reduced maps whose graphs are not even 2-connected. An example of such a map on the torus is given in Fig. 1.

The reason why we are interested in reduced maps is the following.

Lemma 2.2. Let $M$ be a map on a flat surface which has a convex representation. Then $M$ is reduced.

Proof. Being reduced is a topological invariant, so we may assume that $M$ is convex. It is clear that $M$ has no vertices of degree 1 since a face containing such a vertex cannot be convex. Suppose now that $G$ contains a cycle $C$ and disk $D$ satisfying (R2). Consider $D$ and its interior angles $\alpha_{1}, \ldots, \alpha_{k}$. By (R2) it follows that all of these angles, except at most two of them, are larger or equal to $\pi$. Thus,

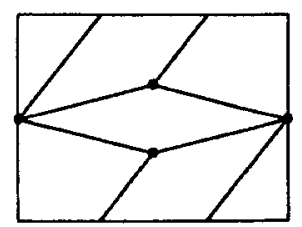

Fig. 1. A reduced toroidal map whose graph is not 2-connected. 
$\sum_{i=1}^{k} \alpha_{i}>(k-2) \pi$. On the other hand, the Gauss-Bonnet theorem (see [1]) implies that $\sum_{i=1}^{k} \alpha_{i}=(k-2) \pi$ since $D$ is a flat disk. This is a contradiction. The exclusion of the remaining possibility $(\mathrm{R} 3)$ is similar.

\section{Convex Representations in a Disk}

By a theorem of Stein [12] and Tutte [15] every 3-connected planar graph has a convex representation in the plane. Moreover, Tutte established a necessary and sufficient condition for a 2-connected graph $G$ with a given cycle $C$ to have a convex embedding in the plane such that $C$ bounds the unbounded face and is a convex $|C|$-gon. Tutte's condition is that $G$ is a subdivision of a 2-connected graph $H$ such that every separating set $\{u, v\}$ of $H$ (if any) is contained in the cycle of $H$ corresponding to $C$. This result was generalized by Thomassen [14] to cover the general case where $C$ need not be strictly convex, i.e., it could be a $k$-gon with $k<|C|$.

Let $H$ be a subgraph of $G$. A relative $H$-component is a subgraph of $G$ which is either an edge $e \in E(G) \backslash E(H)$ (together with its endpoints), which has both endpoints in $H$, or it is a connected component of $G-V(H)$ together with all edges (and their endpoints) between this component and $H$. Occasionally, the relative $H$-components are also called bridges of $H$. Each edge of an $H$-component $R$ having an endpoint in $H$ is a foot of $R$. The vertices of $R \cap V(H)$ are the vertices of attachment of $R$. We use the same terminology for maps and their submaps.

Theorem 3.1 [14]. Let $C$ be a cycle of a simple 2-connected planar graph $G$, and let $R$ be a convex $k$-gon $(k \leq|V(C)|)$ in the plane with sides $P_{1}, P_{2}, \ldots, P_{k}$, occurring in the listed order on $R$. A given convex embedding of $C$ into $R$ can be extended to $a$ convex embedding of $G$ such that $R$ bounds the outer face if and only if:

(a) For each vertex $v \in V(G) \backslash V(C)$ of degree at least 3, G contains three paths from $v$ to $C$ which pairwise have only $v$ in common.

(b) No $C$-component of $G$ has all its vertices of attachment in some $P_{i}, 1 \leq i \leq k$.

(c) Any cycle of $G$ which has no edge in common with $C$ has at least three vertices of degree $\geq 3$ in $G$.

A proof of Theorem 3.1 can be found in [14]. It is important that the proof yields a linear-time algorithm for convex drawings of planar graphs. More precisely, there is an algorithm that given $G, C, R$, and an embedding of $C$ into $R$, extends the embedding of $C$ to a convex embedding of $G$ into $\operatorname{int}(R)$, whenever possible, and uses linear time and space. See [2] and [11] for details. In addition to this, appropriate cycles $C$, if any, can be found in linear time [2], [11].

Consider a submap $L$ of $M$. A vertex of degree 3 or more in $L$ is called a main vertex of $L$. The paths in $L$ joining pairs of main vertices and having all internal vertices of degree 2 in $L$ are called branches of $L$. Each branch $e$ gives rise to two sides of $e$ which correspond to the "left" and "right" (or "upper" and "lower") side of the branch on the surface. Note that since $L$ is a map, the sides are well 
defined. For example, if $L$ is homeomorphic to map $M_{1}$ of Fig. 2(b), it has six sides. Let $Q$ be a relative $L$-component and $\sigma$ a side of $L$. We say that $Q$ is attached to the side $\sigma$ if all feet of $Q$ attach to $L$ at the side of the branch corresponding to $\sigma$, including the possibility of the attachment at an endpoint of the branch (as far as the side is correct). In the same way we define when $Q$ is attached to a set of sides.

Suppose that $L$ is a convex map. If $F$ is a face, then we can speak of the sides of the convex polygon determined by $F$. Note that the number of sides of the convex polygon determined by $F$ can be smaller than the number of branches of $L$ on the boundary of $F$. See, e.g., Fig. 3(a) where the facial polygon has four sides while there are six sides of branches appearing on its boundary.

As a corollary to Theorem 3.1 we get the following important lemma.

Lemma 3.2. Let $L$ be a submap of a reduced map $M$ on a flat surface $S$. Suppose that $L$ is convex and that no relative $L$-component of $M$ is attached to a single side of the convex polygon determined by the face of $L$ it lies in. Then $L$ can be extended to a convex representation of $M$.

Proof. Consider a face $F$ of $L$. Suppose that $F$ is an open convex $k$-gon. Let $M_{F}$ be the map in the disk obtained as follows. First take a convex $k$-gon $D$ (in the plane) with the same angles and side lengths as there are in $F$. Add vertices of $L$ on the boundary of $D$ in exactly the same way as they appear on the boundary of $F$. In the case when there is a multiple occurrence of a vertex on $\partial F$, choose a new copy of the vertex for each of the occurrences. Finally, add in $D$ the relative $L$-components of $M$ lying in $F$ so that they are embedded in exactly the same way as in $F$. We claim that $M_{F}$ satisfies the conditions of Theorem 3.1. (Once we verify this claim, the proof will be evident.)

By construction, the outer boundary of $M_{F}$ is a cycle. Denote it by $C$. The graph of $M_{F}$ is simple since a loop or a pair of parallel edges necessarily bound a disk (also in $M$ ) and this violates (R2) or (R3) (in case when a parallel pair joins two vertices of $C$ which represent the same vertex of $L$ ). It also follows from (R2) that $M_{F}$ has no cut-vertices and is therefore 2-connected.

It remains to check properties (a)-(c) of Theorem 3.1. Condition (b) is among the assumptions. To check (a), suppose that there is a vertex $v \in V(M) \backslash V(C)$ of degree 3 or more in $M_{F}$ such that there are at most two paths from $v$ to $C$. By Menger's theorem there are vertices $a, b$ in $M_{F}$ different from $v$ such that any path from $v$ to $C$ uses one of them. Let $Q$ be the submap of $M_{F}$ consisting of all edges which are contained in those paths starting at $v$ which do not contain $a$ or $b$ as an interior vertex. Note that $V(Q) \cap V(C)=\{a, b\} \cap V(C) . Q$ is a connected plane subgraph of $M_{F}$. Since $v$ is of degree at least 3 in $Q$ and $Q$ contains at most two vertices of degree 1 (by (R1) the only candidates are $a$ and $b$ ), $Q$ contains a cycle. Let $W$ be the facial walk corresponding to the outer face of $Q$. Since $Q$ contains a cycle, there is a subwalk $W^{\prime}$ of $W$ which is a cycle of the graph. If possible, choose $W^{\prime}$ in such a way that it does not contain both vertices $a$ and $b$. In any case, $W^{\prime}$ either gives rise to a contractible cycle in $M$ contradicting (R2) or to a pair of homotopic cycles in $M$ that contradict (R3). 
It remains to check that each cycle $C^{\prime}$ in $M_{F}$ such that $E\left(C^{\prime}\right) \cap E(C)=\varnothing$ has at least three vertices of degree greater or equal to 3 . Suppose this is not true. The cycle $C^{\prime}$ bounds a disk in $M_{F}$ and hence it also bounds a disk in $M$, although it may change there into a union of two homotopic cycles. The required property is now apparent since otherwise we would have a cycle (or two cycles) in $M$ violating (R2) or (R3).

We need another lemma concerning relative $L$-components attached to a single side of a branch of $L$. Such a relative component is said to be local. Those relative $L$-components which are not local are called global. Note that a relative $L$ component attached to a single branch $e$ of $L$ is not local if it is attached to both sides of $e$. A map $M^{\prime}=\left(G^{\prime}, S^{\prime}\right)$ is homeomorphic to the map $M=(G, S)$ if there is a homeomorphism $h: S^{\prime} \rightarrow S$ whose restriction to $G^{\prime}$ is a 1-1 map onto $G \subset S$ (a graph homeomorphism).

Lemma 3.3. Let $L$ be a submap of a reduced map $M$. Then there is a submap $L^{\prime \prime}$ of $M$ which is homeomorphic to $L$ and such that there are no local relative $L^{\prime \prime}$-components and such that $L^{\prime \prime}$ has the same main vertices as $L$. Moreover, the homeomorphism between $L$ and $L^{\prime \prime}$ can be chosen to be the identity on the set of main vertices of $L$. Given $L$, then $L^{\prime \prime}$ can be found in time which is linear in the number of edges of $M$.

Proof. In addition to the properties stated in the lemma, every branch of $L^{\prime \prime}$ will be contained in the corresponding branch of $L$ together with the local relative $L$-components attached to one or the other of its sides. First we construct a submap $L^{\prime}$ as follows. Fix a side $\sigma$ of $L$. Determine all local relative $L$-components attached at $\sigma$. We can perform this operation for all sides $\sigma$ at once in linear time by a properly modified version of the breadth-first search (or depth-first search). The local relative $L$-components attached to $\sigma$ are partially ordered: $Q^{\prime} \leq Q$ if $Q$ together with its segment of attachment to $\sigma$ (=the segment on $\sigma$ between the two vertices of attachment of $Q$ which are the most apart on $\sigma$ ) bounds a disk which contains $Q^{\prime}$. For each maximal (with respect to the presented partial order) relative $L$-component attached at $\sigma$, replace the interval of attachment of this relative $L$-component by the outer path in this relative component. Note that the maximal components are easy to discover from the rotation system of the map. Repeat the procedure for one of the sides of each branch of $L$, and let $L^{\prime}$ be the obtained submap of $M$. Note that there are no local relative $L^{\prime}$-components attached to the sides that have been processed above. Since $M$ is reduced, every local relative $L^{\prime}$-component overlaps on the other side with another relative $L^{\prime}$-component. By the above, this component is not local. Thus, the repetition of the above procedure with $L^{\prime}$, using the other sides of the branches, gives rise to a submap $L^{\prime \prime}$ of $M$ without local relative $L^{\prime \prime}$-components. Note that the local relative components have changed when $L$ was replaced by $L^{\prime}$. Therefore we need to perform the operation of their construction once again.

The time needed in the above algorithm for any one of the sides is linear in the number of edges in the corresponding branch together with all local relative $L$-components attached at this side. Therefore the total time is linear. 


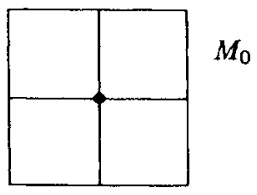

(a)

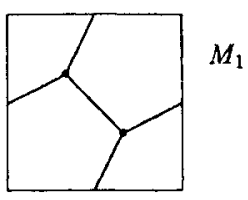

(b)

Fig. 2. Toroidal maps $M_{0}$ and $M_{1}$.

\section{The Torus}

Denote by $M_{0}$ and $M_{1}$ the toroidal maps represented in Fig. 2(a) and (b), respectively.

Lemma 4.1. Let $M$ be a toroidal map. Then $M$ contains a submap $L$ which is homeomorphic to one of the maps $M_{0}$ or $M_{1}$ of Fig. 2. Such a submap can be found in linear time.

Proof. The lemma is quite obvious. Let us just describe a way we can find the required submap $L$. We first find a spanning tree $T$ of the map by using the Depth First Left Second Search. This means that in the DFS, whenever we have a choice of more than one edge to consider, we always take the one which is the leftmost untraversed according to the rotation system of the map. At the same time we make a circular list of the edges in the cotree in the order as they were found during the tree construction, and such that each edge appears twice in the list, once for each of its ends. It is easy to see that the order of edges coincides with the circular orders obtained as the rotation system when the spanning tree $T$ is contracted to a point. Let $e$ and $f$ be edges which are interlaced in this list, i.e., their order is $\ldots, e, \ldots, f, \ldots, e, \ldots, f \ldots$ (It is easy to see that if there is no such pair, then the map is not toroidal.) Let $C_{e}$ and $C_{f}$ be the fundamental cycles of $e$ and $f$, respectively. Then $C_{e} \cup C_{f}$ is the required map $L$ homeomorphic to $M_{0}$ or to $M_{1}$.

It may not seem very obvious how we can choose in linear time an interlacing pair $e, f$. A simple way to do this is to start building a stack by traversing the list once around, and adding edges met during the traversal on the top of the stack when the edge is met for the first time. Meeting the edge for the second time, we check if the top element in the stack is the same edge. If yes, the edge is removed from the stack and the traversal is continued. If not, then we have another edge at the top. It is easy to see that these two edges interlace in our list.

Theorem 4.2. A toroidal map is equivalent to a convex map on the flat torus if and only if it is reduced.

Proof. The "only if" part follows by Lemma 2.2, and to prove the "if" part we use Lemmas 4.1, 3.2, and 3.3. 
Corollary 4.3. Let $M$ be a toroidal map whose graph is 3-connected. Then $M$ has a convex representation in the flat torus.

Proof. Obvious by Theorem 4.2 and Proposition 2.1.

It is important that our proof of Theorem 4.2 yields a linear-time algorithm for convex drawings of toroidal maps. Our algorithm is carried out in four steps:

\section{Algorithm TOROIDAL CONVEX EMBEDDING}

(1) Find a submap $L$ homeomorphic to $M_{0}$ or $M_{1}$ (see Lemma 4.1 and its proof).

(2) Get rid of the local relative $L$-components (see Lemma 3.3 and its proof).

(3) Construct $M_{F}$ (see the proof of Lemma 3.2) where $F$ denotes the face of $L$. It is obvious that $M_{F}$ can be constructed in linear time.

(4) Find a convex representation of $M_{F}$. This can be done in linear time as described in [2]. See also [11].

It is worth mentioning that instead of taking the map $M_{1}$ of Fig. 2(b), we can take the map $M_{1}^{\prime}$ of Fig. 3(a) to start with. The advantage is that the final drawing will be nicer since it will either be represented in a square (if $L$ is homeomorphic to $M_{0}$ ) or in a rhombus as shown in Fig. 3(b), with the obvious identifications giving rise to the flat torus.

To get a realization of this form it has to be shown that in the case when we need to use the submap $L$ homeomorphic to $M_{1}^{\prime}$ we can choose $L$ so that any relative $L$-component attached at the sides $\alpha$ and $\beta$ (or $\alpha^{\prime}$ and $\beta^{\prime}$, respectively) is indeed local at a single one of these sides. To reach this, we modify step (2) of the above algorithm as follows. We first change the map $L$ obtained in step (1) so that it either becomes homeomorphic to $M_{0}$ or it is homeomorphic to $M_{1}^{\prime}$ and every relative $L$-component which is attached to $\alpha$ and $\beta$ (or $\alpha^{\prime}$ and $\beta^{\prime}$, respectively) is local. Determine all global relative $L$-components which are attached to the sides $\alpha$ and $\beta$ only. Such relative $L$-components are nested and we find the "outermost" relative $L$-component $Q$ among them. Using $Q$, we change $L$ by replacing the part of the branch $\beta$ between the end of $\beta$ and the rightmost

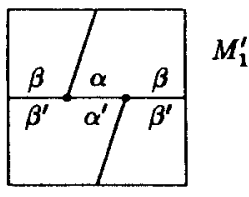

(a)

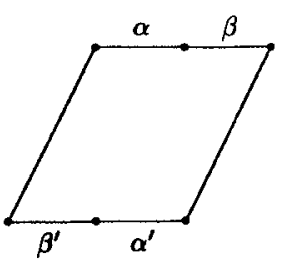

(b)

Fig. 3. The map $M_{1}^{\prime}$ and the corresponding quadrangle. 
attachment of $Q$ with the outer path in $Q$. Now repeat the same step for $\alpha^{\prime}$ and $\beta^{\prime}$ (in the new map). It is easy to see that after these two steps, there are no more global relative components attached only to $\alpha$ and $\beta$ (or $\alpha^{\prime}$ and $\beta^{\prime}$, respectively), and that these operations can be performed in linear time.

\section{The Klein Bottle}

Theorem 5.1. Every reduced map on the Klein bottle has a convex representation on the flat Klein bottle.

Proof. The proof is basically the same as in case of the torus. Relying on the results of Sections 2 and 3 it suffices to find the appropriate convex submap $L$ of the given reduced map $M$ on the Klein bottle. In order to see what are the unavoidable submaps, and how to get them (in linear time), we describe the algorithm. As in the case of the torus we use the Depth First Left Second Search to construct a spanning tree $T$ of $M$. Since the Klein bottle is nonorientable the situation is a little more complex in this case. Note that the map can be given by means of local rotations at the vertices together with an assignment $\lambda: E(M) \rightarrow$ $\{-1,1\}$ (an embedding scheme, see, e.g., [7] or [10]). The meaning of $\lambda$ is the following. Traversing an edge with $\lambda(e)=1$ the two local rotations at the endvertices of $e$ are consistent (as on the surface), and in the case $\lambda(e)=-1$ they are nonconsistent. For any vertex $v$ we may change $\lambda$ on all edges incident with $v$ and at the same time reverse the local rotation at $v$, and we get an equivalent embedding scheme which represents the same map. Call this operation the switch at $v$. Constructing the DFS spanning tree we use the switch operation at the vertex $v$ when we add the vertex $v$ to the tree if the edge $e$ on which we came to $v$ has $\lambda(e)=-1$. It follows that at the end all edges $e$ in the obtained spanning tree $T$ have $\lambda(e)=1$.

During the construction of $T$ we build the circular list of cotree edges as in the case of the torus. Define the matrix $A=\left(a_{e f}\right), e, f \in E(M) \backslash E(T)$, indexed by the cotree edges, where

$$
a_{e f}= \begin{cases}1 & \text { if } e=f \quad \text { and } \quad \lambda(e)=-1 \\ 1 & \text { if } e \neq f \quad \text { and } e \text { and } f \text { interlace in the list } \\ 0 & \text { otherwise. }\end{cases}
$$

It is shown in [7] that the rank of $A$ over the field $G F(2)$ is equal to 2. Moreover, since the surface is nonorientable, at least one diagonal entry is equal to 1 . It follows that there are cotree edges $e$ and $f$ such that their $2 \times 2$ submatrix of $A$ is equal to

$$
A_{\text {ef }}=\left[\begin{array}{ll}
1 & 1 \\
1 & 0
\end{array}\right]
$$




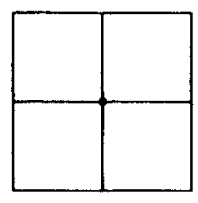

(a)

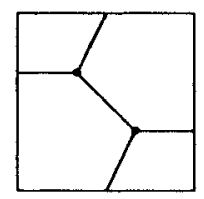

(b)

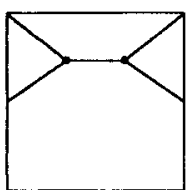

(c)

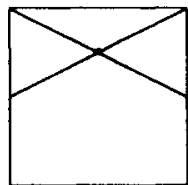

(d)

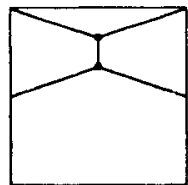

(e)

Fig. 4. Unavoidable submaps on the Klein bottle.

or to

$$
A_{e f}=\left[\begin{array}{ll}
1 & 0 \\
0 & 1
\end{array}\right]
$$

In the first case, the union of the fundamental cycles of $e$ and $f$ determines a submap homeomorphic to the map in either Fig. 4(a) or (b). In the second case, we get Fig. 4(c), (d), or (e).

To find appropriate edges $e$ and $f$ it is not necessary to compute the whole overlap matrix $A$. All the necessary information is already in the constructed doubly linked list of the cotree edges. We can find the required pair of edges $e$ and $f$ in linear time as follows. An edge $e$ with $\lambda(e)=1$ is said to be orientation preserving and it is orientation reversing if $\lambda(e)=-1$. First we try to find orientation-reversing cotree edges $e, f$ which do not interlace (the submatrix (2)). For each orientation-reversing edge $e$ in the list, we determine the number of orientation-reversing edges between $e$ and the next occurrence of $e$ in the list. (This can be achieved in linear time as follows. One of the edges is selected as the first one. By traversing the list, we determine, for each cotree edge $e$, the number of orientation-reversing edges in the list from the first one up to $e$. By another traversal of the list, we can determine, for each edge $e$ in the list, the number of orientation-reversing edges between $e$ and its next occurrence cyclically in the given direction.) Select the element in the list where this number is minimal. It is easy to see that either there is an orientation-reversing edge $f$ which does not occur in the part of the list between the selected element and its second occurrence (in which case we are done), or the orientation-reversing edges interlace evenly, i.e., the sublist of orientation reversing edges is $\left(e_{1}, e_{2}, \ldots, e_{k}, e_{1}, e_{2}, \ldots, e_{k}\right)$ in which case there is no solution of this type. In the latter case, we have to look for an orientation-reversing edge $e_{i}$ which interlaces with an orientation-preserving edge 
$f$ (their submatrix of $A$ is given in (1)). Find an orientation-preserving edge $f$ such that each of the two sublists between the occurrences of $f$ contains an orientationreversing edge. (A linear-time procedure for this task is similar to above.) It is easy to see that such an edge necessarily exists, and that it interlaces with one of the $e_{i}(1 \leq i \leq k)$.

It is worth mentioning that the above proof yields a linear-time algorithm for convex drawings of reduced maps on the Klein bottle.

\section{The Cylinder and the Möbius Band}

We represent the maps on the cylinder and the Möbius band by a unit square with the top and the bottom forming the boundary of the surface, and the left and the right side identified (after a twist for the Möbius band). To mark that the boundary is covered by edges, it is drawn thicker.

Theorem 6.1. Every reduced map on the cylinder has a convex representation on the flat cylinder.

Proof. Let $M$ be a reduced map on the cylinder. It is obvious that $M$ contains a submap homeomorphic to the map $L$ represented in Fig. 5(a). By Lemma 3.2, it suffices to see that $L$ can be chosen such that there are no local relative $L$-components. However, this follows by Lemma 3.3 .

Theorem 6.2. Every reduced map on the Möbius band has a convex representation on the flat Möbius band.

The proof is the same as for Theorem 6.1. The only difference is that we have three unavoidable submaps shown in Fig. 5(a), (b), and (c). Note that the side identifications are meant to give the Möbius band in this case.

Linear-time algorithms for the case of the cylinder and the Möbius band are similar as in the case of the torus or the Klein bottle. We omit the details.

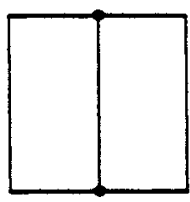

(a)

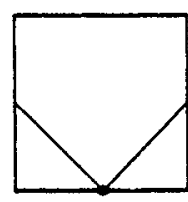

(b)

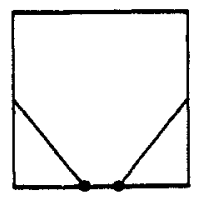

(c)

Fig. 5. Unavoidable submaps on the cylinder and the Möbius band. 


\section{Straight-Line Representations}

A simple corollary to our results in the previous sections is a characterization of maps that admit straight-line representations. These are representations where each edge is locally a straight-line segment.

Theorem 7.1. A map $M$ on a surface $S$ with Euler characteristic 0 admits straight-line representations if and only if it fulfills the following conditions:

(a) Each pair of parallel edges (edges with the same end-vertices) gives rise to a noncontractible cycle on $S$.

(b) No loop of $M$ is contractible.

(c) If $e$ is a loop at the vertex $v$, then no other loop at $v$ is homotopic to $e$.

Moreover, there is a linear-time algorithm to find a straight-line representation of a map satisfying the above conditions.

Proof. The given conditions are necessary (see the proof of Lemma 2.2). Conversely, if $M$ satisfies (a)-(c), then each face can be triangulated so that the obtained map $\tilde{M}$ is reduced. Triangulating can be performed in linear time. Now, the results of the previous sections apply.

A very simple proof of Theorem 7.1 is presented in [8]. A generalization of this result to maps on arbitrary (closed) surfaces can be obtained by using the Andreev-Koebe-Thurston circle-packing theorem. See [9] for details.

\section{References}

1. M. P. do Carmo, Differential Geometry of Curves and Surfaces, Prentice-Hall, Englewood Cliffs, NJ, 1976.

2. N. Chiba, T. Yamanouchi, and T. Nishizeki, Linear algorithms for convex drawings of planar graphs, in Progress in Graph Theory, eds. J. A. Bondy and U. S. R. Murty, Academic Press, Toronto, 1984, pp. 153-173.

3. P. Gritzmann, The toroidal analogue to Eberhard's theorem, Mathematika 30 (1983), $274-290$.

4. J. L. Gross and T. W. Tucker, Topological Graph Theory, Wiley-Interscience, New York, 1987.

5. B. Grünbaum and G. C. Shephard, Tilings and Patterns, Freeman, New York, 1987.

6. P. Mani-Levitska, B. Guigas, and W. E. Klee, Rectifiable $n$-periodic maps, Geom. Dedicata 8 (1979), 127-137.

7. B. Mohar, An obstruction to embedding graphs in surfaces, Discrete Math. 78 (1989), 135-142.

8. B. Mohar, Straight line representations of maps on the torus and other flat surfaces, Preprint, 1991.

9. B. Mohar, A polynomial time circle packing algorithm, Discrete Math. 117 (1993), 257-263.

10. B. Mohar and C. Thomassen, Graphs on surfaces, in preparation.

11. T. Nishizeki and N. Chiba, Planar Graphs: Theory and Algorithms, Annals of Discrete Mathematics, Vol. 32, North-Holland, Amsterdam, 1988.

12. S. K. Stein, Convex maps, Proc. Amer. Math. Soc. 2 (1951), $464-466$.

13. E. Steinitz, Polyeder und Raumeinteilungen, Enzykl. math. Wiss. Part 3AB12 (1922), 1-139. 
14. C. Thomassen, Planarity and duality of finite and infinite graphs, J. Combin. Theory Ser. B 29 (1980), $244-271$.

15 W. T. Tutte, Convex representations of graphs, Proc. London Math. Soc. 10 (1960), 304-320.

16. C. Thomassen, Plane representations of graphs, in Progress in Graph Theory, eds. J. A. Bondy and U. S. R. Murty, Academic Press, Toronto, 1984, pp. 43-69.

Received February 25, 1992, and in revised form May 25, 1993.

Note added in proof. We have learnt recently that a slightly weaker result than our Theorem 4.2 was proved by Thomassen [16, Theorem 5.1]. 